\title{
A complete characterization of $R$-sets in the theory of differentiation of integrals
}

by

\author{
G. A. Karagulyan (Yerevan)
}

\begin{abstract}
Let $\mathcal{R}_{s}$ be the family of open rectangles in the plane $\mathbb{R}^{2}$ with a side of angle $s$ to the $x$-axis. We say that a set $S$ of directions is an $R$-set if there exists a function $f \in L^{1}\left(\mathbb{R}^{2}\right)$ such that the basis $\mathcal{R}_{s}$ differentiates the integral of $f$ if $s \notin S$, and $\bar{D}_{s} f(x)=\limsup _{\operatorname{diam}(R) \rightarrow 0, x \in R \in \mathcal{R}_{s}}|R|^{-1} \int_{R} f=\infty$ almost everywhere if $s \in S$. If the condition $\bar{D}_{s} f(x)=\infty$ holds on a set of positive measure (instead of a.e.) we say that $S$ is a $W R$-set. It is proved that $S$ is an $R$-set (resp. a $W R$-set) if and only if it is a $G_{\delta}$ (resp. a $G_{\delta \sigma}$ ).
\end{abstract}

1. Introduction. For any $s \in[0, \pi / 2)$ we define $\mathcal{R}_{s}$ to be the family of all open rectangles $R$ in $\mathbb{R}^{2}$ that have a side at angle $s$ to the $x$-axis. We say that the basis $\mathcal{R}_{s}$ differentiates the integral of the function $f \in L^{1}\left(\mathbb{R}^{2}\right)$ if

$$
\lim _{\operatorname{diam}(R) \rightarrow 0, x \in R \in \mathcal{R}_{s}} \frac{1}{|R|} \int_{R} f=f(x)
$$

almost everywhere in $\mathbb{R}^{2}$, where $d(R)$ is the diameter of $R$. According to the well-known theorem of Jessen-Marcinkiewicz-Zygmund [3], $\mathcal{R}_{s}$ differentiates $\int f$ for any $f \in L \log L\left(\mathbb{R}^{2}\right)$. On the other hand, S. Saks [12] constructed an $f \in L^{1}\left(\mathbb{R}^{2}\right)$ such that

$$
\bar{D}_{s} f(x)=\limsup _{\operatorname{diam}(R) \rightarrow 0, x \in R \in \mathcal{R}_{s}} \frac{1}{|R|} \int_{R} f=\infty \quad \text { everywhere. }
$$

In view of this A. Zygmund posed the following problem (see [2]): for a given $f \in L^{1}\left(\mathbb{R}^{2}\right)$, is it possible to find a direction $s$ such that $\mathcal{R}_{s}$ differentiates $\int f$ ? J. Marstrand in [7] gave a negative answer to this question, proving

TheOREM (J. Marstrand). There exists a function $f \in L^{1}\left(\mathbb{R}^{2}\right)$ such that $\bar{D}_{s} f(x)=\infty$ almost everywhere for any $s$.

2000 Mathematics Subject Classification: Primary 42B25.

Key words and phrases: differentiation of integrals, maximal functions, Zygmund's problem. 
Different generalizations of this result have been obtained by J. El Helou [1], A. M. Stokolos [13], B. López Melero [6] and G. G. Oniani [9]. A. M. Stokolos [13] extended Marstrand's theorem to higher dimensions. In [6] and [9] the same problem is considered for general translation invariant differentiation bases.

We say that a set $S \subset[0, \pi / 2)$ is an $R$-set if there exists a function $f \in L^{1}\left(\mathbb{R}^{2}\right)$ such that the basis $\mathcal{R}_{s}$ differentiates $\int f$ whenever $s \in[0, \pi / 2) \backslash S$, and $\bar{D}_{s} f(x)=\infty$ almost everywhere if $s \in S$. If $\bar{D}_{s} f(x)=\infty$ on a set of positive measure (instead of a.e.), we call $S$ a $W R$-set (weak $R$-set). In this terminology, Marstrand's theorem asserts that $[0, \pi / 2)$ is an $R$-set. A. M. Stokolos [14] proved the existence of an everywhere dense $W R$-set which is not the whole $[0, \pi / 2)$. G. Lepsveridze [4], [5] proved that any finite set is an $R$-set and any countable set is contained in some $W R$-set of measure zero. G. G. Oniani [9], generalizing this result, proved that any countable set is contained in some $R$-set of measure zero.

The definition of $R$-sets first appeared in the paper [8] by G. G. Oniani, who posed the problem of characterization of all $R$-sets. In particular, he asked if there exists an $R$-set of positive measure and whether any interval is an $R$-set. In the same paper Oniani shows that any $R$-set is a $G_{\delta}$ in $[0, \pi / 2)$, i.e.

$$
G=\left(\bigcap_{k=1}^{\infty} G_{k}\right) \cap[0, \pi / 2)
$$

where $G_{n}$ are open sets, and conversely, if a $G_{\delta}$-set is countable, then it is an $R$-set. These results characterize the countable $R$-sets. We note that any countable $G_{\delta}$-set is nowhere dense. So in [8] Oniani also constructed an $R$-set of second category. These problems are also stated in Oniani's monograph [9], and the higher dimensional case of the problem is investigated in [10] and [11].

The following theorems give a complete characterization of general $R$-sets and $W R$-sets.

Theorem 1. A set $S \subset[0, \pi / 2)$ is an $R$-set if and only if it is a $G_{\delta}$.

TheOrem 2. A set $S \subset[0, \pi / 2)$ is a WR-set if and only if it is a $G_{\delta \sigma}$.

The necessity of Theorem 1 is proved by Oniani in [8]. We briefly sketch that proof. If $S$ is an $R$-set, then there exists $f \in L^{1}$ such that (1.1) holds if $s \in[0, \pi / 2) \backslash S$, and $\bar{D}_{s} f(x)=\infty$ a.e. if $s \in S$. For any $n \in \mathbb{N}$ define

$$
U_{n}=\left\{s \in[0, \pi / 2):\left|\left\{x \in B(n): M_{s} f(x)>n\right\}\right|>|B(n)|-2^{-n}\right\},
$$

where $B(n)=\left\{x \in \mathbb{R}^{2}:\|x\| \leq n\right\}$ and the maximal function $M_{s} f$ is defined in Section 2. It is easy to check that $U_{n}=G_{n} \cap[0, \pi / 2)$, where $G_{n}$ are open 
sets and

$$
\left\{s \in[0, \pi / 2): \bar{D}_{s} f(x)=\infty \text { a.e. }\right\}=\bigcap_{n} U_{n}=\left(\bigcap_{n} G_{n}\right) \cap[0, \pi / 2),
$$

i.e. it is a $G_{\delta}$-set in $[0, \pi / 2)$, which proves one direction of Theorem 1.

To prove the necessity of Theorem 2 it is enough to prove that for any $f \in L^{1}\left(\mathbb{R}^{2}\right)$ the set

$$
G_{f}=\left\{s \in[0, \pi / 2):\left|\left\{x \in \mathbb{R}^{2}: \bar{D}_{s} f(x)=\infty\right\}\right|>0\right\}
$$

is a $G_{\delta \sigma}$. Define

$$
U_{n m}=\left\{s \in[0, \pi / 2):\left|\left\{x \in B(n): M_{s} f(x)>m\right\}\right|>0\right\}, \quad n, m=1,2, \ldots,
$$

where $B(n)$ and $M_{s} f$ are defined in Section 2. It is clear that the $U_{n m}$ are open sets in $[0, \pi / 2)$ and

$$
G_{f}=\bigcup_{n} \bigcap_{m} U_{n m}
$$

To show the last equality it suffices to check the following relations:

$$
\begin{aligned}
s \in G_{f} & \Leftrightarrow\left|\left\{x \in \mathbb{R}^{2}: \bar{D}_{s} f(x)=\infty\right\}\right|>\alpha>0 \\
& \Leftrightarrow \exists n \text { such that }\left|\left\{x \in B_{n}: \bar{D}_{s} f(x)=\infty\right\}\right|>\alpha \\
& \Leftrightarrow \exists n \text { such that } s \in \bigcap_{m} U_{n, m} \Leftrightarrow s \in \bigcup_{n} \bigcap_{m} U_{n, m} .
\end{aligned}
$$

Hence the set $G_{f}$ is a $G_{\delta \sigma}$.

We shall prove the sufficiency of both theorems invoking the probabilistic independence of sets similarly to the original approach of J. Marstrand [7]. This idea is applied in Lemma 1. Of course, we use also Bohr's construction displayed in Saks's classical counterexample. It is important that the function constructed in the proof is not nonnegative, in contrast to all the results stated above. This argument gives more freedom in the construction to ensure differentiability of the integral along some directions. So our method differs from the others, because we essentially use interference of positive and negative values of a function in integrals, which is displayed in Lemmas 2 and 3.

2. Notations and lemmas. The basis $\mathcal{R}_{s}$ can be defined for any $s \in$ $[0,2 \pi]$. We note that $\mathcal{R}_{s}=\mathcal{R}_{t}$ if $s=t \bmod \pi / 2$. In fact $\bigcup_{s \in[0, \pi / 2)} \mathcal{R}_{s}$ is the family of all rectangles in the plane.

If $n \in \mathbb{N}$ and $c=\left(c_{1}, c_{2}\right)$, then for any set $A \subset \mathbb{R}^{2}$ we define

$$
\begin{aligned}
\operatorname{dil}_{n} A & =\left\{x=\left(x_{1}, x_{2}\right) \in \mathbb{R}^{2}: n x=\left(n x_{1}, n x_{2}\right) \in A\right\}, \\
c+A & =\left\{x \in \mathbb{R}^{2}: x=c+a, a \in A\right\} .
\end{aligned}
$$

We let $Q_{0}=[-1 / 2,1 / 2) \times[-1 / 2,1 / 2)$ and for any $n \in \mathbb{N}, k=\left(k_{1}, k_{2}\right) \in \mathbb{Z}^{2}$ define $Q_{k}^{n}=\operatorname{dil}_{n}\left(k+Q_{0}\right)$. For a fixed $n$ the family $\left\{Q_{k}^{n}: k \in \mathbb{Z}^{2}\right\}$ is a 
partition of the plane into squares with side lengths $1 / n$. Occasionally we write simply $Q_{k}$ for $Q_{k}^{1}$.

We denote by $\operatorname{rot}_{s} A$ the rotation of the set $A \subset \mathbb{R}^{2}$ about $(0,0)$ by angle $s$. Let

$$
\begin{aligned}
B(\varepsilon) & =\left\{x \in \mathbb{R}^{2}:\|x\|=\sqrt{x_{1}^{2}+x_{2}^{2}} \leq \varepsilon\right\}, \\
\Gamma_{s}(\varepsilon) & =\operatorname{rot}_{s}\left\{x=\left(x_{1}, x_{2}\right):\left|x_{2}\right|<\varepsilon\right\} .
\end{aligned}
$$

The notation $s^{\perp}$ stands for the direction $s+\pi / 2$. For any direction $s$ define $\operatorname{mes}_{s} A$ to be the linear Lebesgue measure of the projection of $A$ on the line parallel to $s^{\perp}$.

For any measurable set $A \subset \mathbb{R}^{2}$ we define

$$
\operatorname{mes}^{*} A=\sup _{k \in \mathbb{Z}^{2}}\left|A \cap Q_{k}\right|, \quad \operatorname{mes}_{*} A=\inf _{k \in \mathbb{Z}^{2}}\left|A \cap Q_{k}\right| .
$$

For $0<\delta<\mu \leq \infty$ we define $\mathcal{R}_{s}^{[\delta, \mu)}$ to be the family of rectangles $R=$ $R_{1} \times R_{2} \in \mathcal{R}_{s}$ with $\delta \leq\left|R_{1}\right|,\left|R_{2}\right|<\mu$ and we let $\mathcal{R}_{s}^{\delta}$ be the rectangles from $\mathcal{R}_{s}$ with $\left|R_{1}\right|=\left|R_{2}\right|=\delta$. Define

$$
M_{s}^{[\delta, \mu)} f(x)=\sup _{R \in \mathcal{R}_{s}^{[\delta, \mu)}} \frac{1}{|R|}\left|\int_{R} f(x) d x\right| .
$$

If $\delta=0$ and $\mu=\infty$ we write simply $M_{s} f(x)$. We say that $A \subset \mathbb{R}^{2}$ is a $\delta$-set if it is a union of mutually disjoint rectangles from $\mathcal{R}_{s}^{[\delta, \infty)}$. The following lemma contains the main idea of the proof of Marstrand's theorem.

Lemma 1. Let $0<\delta_{t}<1, t=1, \ldots, T$, and let $A_{t} \subset \mathbb{R}^{2}$ be $\delta_{t}$-sets with $\operatorname{mes}_{*} A_{t}>12 \delta_{t}, t=1, \ldots, T$. Then for any sequence $\left\{n_{t}\right\}$ of integers with $n_{1}=1, n_{t+1}>4 n_{t} / \delta_{t}$, we have

$$
\operatorname{mes}_{*}\left(\bigcup_{t=1}^{T} \operatorname{dil}_{n_{t}} A_{t}\right)>1-\left(1-\frac{\operatorname{mes}_{*} A_{t}}{32}\right)^{T} .
$$

Proof. First we prove that if $B$ is a $\delta$-set with $\operatorname{mes}_{*} B>12 \delta, m, n \in \mathbb{N}$ and $n>(4 / \delta) m$, then there exists a set $\widetilde{B}$ such that

(1) $\widetilde{B} \subset \operatorname{dil}_{m} B$,

(2) for any $k \in \mathbb{Z}^{2}$ the set $\widetilde{B} \cap Q_{k}^{m}$ is a union of squares $Q_{j}^{n}$,

(3) the values $\left|\widetilde{B} \cap Q_{k}^{m}\right|$ are equal for different $k \in \mathbb{Z}^{2}$,

(4) $\operatorname{mes}_{*} \widetilde{B}>\frac{1}{32} \operatorname{mes}_{*} B$.

We note that any rectangle $R \in \mathcal{R}_{s}^{[\delta, \infty)}$ is a union of rectangles from $\mathcal{R}_{s}^{\delta}$. So we have $\operatorname{dil}_{m} B=\bigcup_{i} R_{i}$ where $R_{i} \in \mathcal{R}_{s}^{\delta / m}$. Set

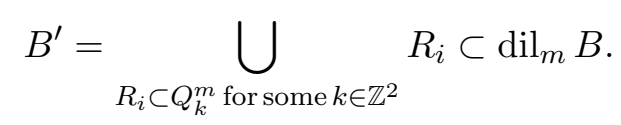


We have diam $R_{i}=\delta \sqrt{2} / m$. So if $R_{i} \not \subset Q_{k}^{m}$ for some $k \in \mathbb{Z}^{2}$, then $R_{i} \cap \widetilde{Q}_{k}^{m}$ is empty, where $\widetilde{Q}_{k}^{m}$ is the square concentric with $Q_{k}^{m}$ with side lengths $(1-2 \delta \sqrt{2}) / m$. Hence we get

$$
\begin{aligned}
\left|B^{\prime} \cap Q_{k}^{m}\right| & >\left|\operatorname{dil}_{m} B \cap Q_{k}^{m}\right|-\left|Q_{k}^{m} \backslash \widetilde{Q}_{k}^{m}\right| \\
& =\left|\operatorname{dil}_{m} B \cap Q_{k}^{m}\right|-\frac{1}{m^{2}}\left(4 \delta \sqrt{2}-8 \delta^{2}\right) \\
& >\left|\operatorname{dil}_{m} B \cap Q_{k}^{m}\right|-\frac{6 \delta}{m^{2}}=\frac{1}{m^{2}}\left|B \cap Q_{k}^{1}\right|-\frac{6 \delta}{m^{2}} \\
& \geq \frac{1}{m^{2}}\left(\operatorname{mes}_{*} B-6 \delta\right)>\frac{\operatorname{mes}_{*} B}{2 m^{2}} .
\end{aligned}
$$

Using the Besicovitch theorem on covering by squares (see [2, p. 10]), we may choose a subfamily $\left\{R_{i}^{\prime}\right\}$ of $\left\{R_{i}\right\}$ such that the $R_{i}^{\prime}$ are pairwise disjoint and

$$
\left|\bigcup_{R_{i}^{\prime} \subset Q_{k}^{m}} R_{i}^{\prime}\right| \geq \frac{1}{4}\left|\bigcup_{R_{i} \subset Q_{k}^{m}} R_{i}\right| \quad \text { for any } k \in \mathbb{Z}^{2} .
$$

Therefore, setting

$$
B^{\prime \prime}=\bigcup R_{i}^{\prime} \subset B^{\prime} \subset \operatorname{dil}_{m} B,
$$

by (2.2) and (2.3) we have

$$
\left|B^{\prime \prime} \cap Q_{k}^{m}\right|>\frac{\operatorname{mes}_{*} B}{8 m^{2}}, \quad k \in \mathbb{Z}^{2} .
$$

Using simple geometry, one can easily check that if $R \in \mathcal{R}_{s}^{\delta / m}$ and $n>4 m / \delta$, then

$$
\left|\bigcup_{Q_{j}^{n} \subset R} Q_{j}^{n}\right|>\frac{1}{4}|R| .
$$

So, by (2.4), for $n>4 m / \delta$ we have

$$
\left|\bigcup_{Q_{j}^{n} \subset B^{\prime \prime} \cap Q_{k}^{m}} Q_{j}^{n}\right|>\frac{1}{4}\left|B^{\prime \prime} \cap Q_{k}^{m}\right|>\frac{\operatorname{mes}_{*} B}{32 m^{2}} .
$$

Removing some squares $Q_{j}^{n}$ from the left union we can get a set $\widetilde{B} \subset B^{\prime \prime}$ which is again a union of squares $Q_{j}^{n}$ and in addition all the sets $\widetilde{B} \cap Q_{k}^{m}$ consist of the same number of squares $Q_{j}^{n}$ and

$$
\left|\widetilde{B} \cap Q_{k}^{m}\right| \geq \frac{\operatorname{mes}_{*} B}{32 m^{2}}, \quad k \in \mathbb{Z}^{2} .
$$

Clearly, $\widetilde{B}$ satisfies conditions (1)-(4) above.

Taking $n=n_{t+1}, m=n_{t}, B=A_{n_{t}}, t=1, \ldots, T$, we get sets $\widetilde{A}_{t}$, $t=1, \ldots, T$, such that

(1) $\widetilde{A}_{t} \subset \operatorname{dil}_{n_{t}} A_{t}$, 
(2) $\widetilde{A}_{t} \cap Q_{k}^{n_{t}}$ is a union of squares $Q_{j}^{n_{t+1}}$ for any $k \in \mathbb{Z}^{2}$,

(3) the values $\left|\widetilde{A}_{t} \cap Q_{k}^{n_{t}}\right|$ are equal for different $k \in \mathbb{Z}^{2}$,

(4) $\operatorname{mes}_{*} \widetilde{A}_{t}>\operatorname{mes}_{*} A_{t} / 32$.

From conditions (2), (3) it follows that for fixed $k \in \mathbb{Z}^{2}$ the sets $\widetilde{A}_{t} \cap Q_{k}$, $t=1, \ldots, T$, are probabilistically independent. Then by (1) and (4),

$$
\begin{aligned}
\operatorname{mes}_{*}\left(\bigcup_{t=1}^{T} \operatorname{dil}_{n_{t}} A_{t}\right) & \geq \operatorname{mes}_{*}\left(\bigcup_{t=1}^{T} \widetilde{A}_{t}\right)=\left|\bigcup_{t=1}^{T}\left(\widetilde{A}_{t} \cap Q_{k}\right)\right| \\
& =1-\left(1-\operatorname{mes}_{*} \widetilde{A}_{t}\right)^{T}>1-\left(1-\frac{\operatorname{mes}_{*} A_{t}}{32}\right)^{T} .
\end{aligned}
$$

For any line $l \subset \mathbb{R}^{2}$ we denote by $\arg l$ the positive value of the minimal angle between $l$ and the $x$-axis. For two points $\theta, \theta^{\prime} \in \mathbb{R}^{2}$ we denote by $\theta \theta^{\prime}$ the line passing through $\theta$ and $\theta^{\prime}$, and by $\left[\theta, \theta^{\prime}\right]$ the line segment with endpoints $\theta$ and $\theta^{\prime}$.

Lemma 2. Let $0<\varepsilon<1,0<\gamma<\pi / 12$ and set

$$
\theta_{k}=\left(\varepsilon / 2^{k}, \operatorname{sign}(k) \cdot \tan \gamma \cdot \varepsilon / 2^{k}\right), \quad k= \pm 1, \pm 2, \ldots
$$

Then for any rectangle $R \in \mathcal{R}_{s}$ with $3 \gamma<|s|<\pi / 2-3 \gamma$, we have

$$
\left|\sum_{0<|k| \leq m, \theta_{k} \in R} \operatorname{sign}(k)\right| \leq 2, \quad m=1,2, \ldots .
$$

Proof. First we note that if $l$ is a line on the plane, then

$$
l \cap\left[\theta_{k}, \theta_{-k}\right] \neq \emptyset, \quad l \cap\left[\theta_{k+1}, \theta_{-(k+1)}\right] \neq \emptyset
$$

implies

$$
\arg l<3 \gamma
$$

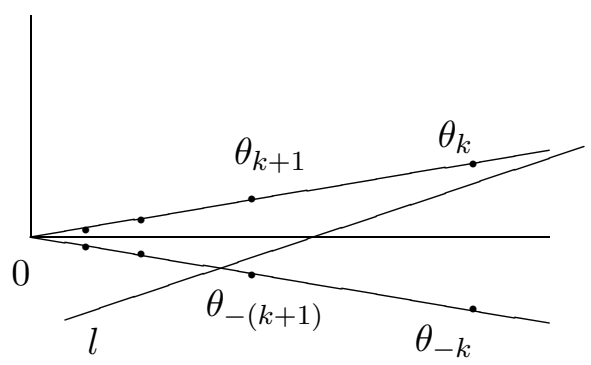

Indeed, using simple geometry, one can check that $\arg \left(\theta_{-k} \theta_{k+1}\right)<3 \gamma$. Hence $\arg l \leq \arg \left(\theta_{-k} \theta_{k+1}\right)<3 \gamma$. Now consider a rectangle

$$
R \in \mathcal{R}_{s}, \quad 3 \gamma<|s|<\pi / 2-3 \gamma .
$$


Let us show that

$$
\text { if } \theta_{n}, \theta_{n+1}, \theta_{n+2} \in R \text {, then } \theta_{-(n+1)} \in R \text {. }
$$

Suppose $\theta_{-(n+1)} \notin R$. Then we find a line $l$ containing a side of $R$ and separating the points $\theta_{n}, \theta_{n+1}, \theta_{n+2}$ from $\theta_{-(n+1)}$. Obviously,

$$
l \cap\left[\theta_{n+1}, \theta_{-(n+1)}\right] \neq \emptyset,
$$

and either $l \cap\left[\theta_{n}, \theta_{-n}\right] \neq \emptyset$, or $l \cap\left[\theta_{n+2}, \theta_{-(n+2)}\right] \neq \emptyset$. So we have (2.7) for $k=n$ or $n+1$ and therefore $\arg l<3 \gamma$, which contradicts (2.8). Similarly

$$
\text { if } \theta_{-n}, \theta_{-(n+1)}, \theta_{-(n+2)} \in R \text {, then } \theta_{n+1} \in R \text {. }
$$

Now let $p$ and $q$ be the numbers of elements of the sets $\left\{1 \leq k \leq m: \theta_{k} \in R\right\}$ and $\left\{-m \leq k \leq-1: \theta_{k} \in R\right\}$ respectively. From (2.9) and (2.10) we conclude $|p-q| \leq 2$, which implies (2.6).

LEMmA 3. For any $0<\varepsilon<1$ and $0<\gamma \leq \pi / 12$ there exists a bounded function $\phi$ on $\mathbb{R}^{2}$ such that

$$
\begin{gathered}
\operatorname{supp} \phi \subset B(\varepsilon), \quad \int_{\mathbb{R}^{2}} \phi(x) d x=0, \quad \int_{\mathbb{R}^{2}}|\phi(x)| d x \leq 1, \\
\int_{\operatorname{rot}_{s}\left(\left[0, x_{1}\right] \times\left[0, x_{2}\right]\right)} \phi(x) d x \geq 1 / 4 \quad \text { if } x_{1}, x_{2} \geq \varepsilon,|s| \leq \gamma, \\
M_{s} \phi(x)<\varepsilon \quad \text { if } x \notin \Gamma_{s}(2 \varepsilon) \cup \Gamma_{s^{\perp}}(2 \varepsilon), 3 \gamma<|s|<\pi / 2-3 \gamma .
\end{gathered}
$$

Proof. Let $\theta=\theta^{+} \cup \theta^{-}$where

$$
\begin{aligned}
& \theta^{+}=\left\{\theta_{k}: k=1,2, \ldots, N\right\}, \\
& \theta^{-}=\left\{\theta_{k}: k=-1,-2, \ldots,-N\right\}, \quad N=\left[10 \varepsilon^{-3}\right]+1,
\end{aligned}
$$

and the $\theta_{k}$ are defined in (2.5). We have

$$
\theta_{k} \in B(\varepsilon / \sqrt{2}) \subset B(\varepsilon), \quad \theta_{k} \in\left\{x: x_{2}=\tan \gamma \cdot x_{1}\right\}, \quad k= \pm 1, \pm 2, \ldots
$$

Define

$$
b_{k}=\left\{x \in \mathbb{R}^{2}:\left|x-\theta_{k}\right|<r\right\}, \quad k= \pm 1, \ldots, \pm N .
$$

For $r>0$ small, the following conditions are satisfied:

(1) $b_{k} \subset B(\varepsilon)$ and they are mutually disjoint,

(2) if $k>0$, then $b_{k}$ is in the upper half-plane, and if $k<0$, in the lower,

(3) any line $l$ with $|\arg l| \geq 3 \gamma$ intersects at most two $b_{k}$.

We define

$$
\phi(x)=\frac{1}{2 \pi N r^{2}} \sum_{k=1}^{N}\left(\mathbb{I}_{b_{k}}(x)+\mathbb{I}_{b_{-k}}(x)\right)
$$


where $\mathbb{I}_{b_{k}}$ is the characteristic function of $b_{k}$. The conditions (2.11) are clear. To show (2.12) we shall use (1) and (2). Fix $x_{1}, x_{2}>\varepsilon$. If $0 \leq s<\gamma$, then

$$
\begin{gathered}
\operatorname{rot}_{s}\left(\left[0, x_{1}\right] \times\left[0, x_{1}\right]\right) \cap b_{k}=\emptyset \quad \text { if }-N \leq k<0, \\
\left|\operatorname{rot}_{s}\left(\left[0, x_{1}\right] \times\left[0, x_{2}\right]\right) \cap b_{k}\right|>\left|b_{k}\right| / 2=\pi r^{2} / 2 \quad \text { if } 0<k \leq N .
\end{gathered}
$$

Therefore

$$
\int_{\operatorname{rot}_{s}\left(\left[0, x_{1}\right] \times\left[0, x_{2}\right]\right)} \phi(x) d x=\frac{1}{2 \pi N r^{2}} \sum_{k=1}^{N} \int_{\operatorname{rot}_{s}\left(\left[0, x_{1}\right] \times\left[0, x_{2}\right]\right)} \mathbb{I}_{b_{k}}(x) d x \geq \frac{1}{4} .
$$

If $-\gamma<s \leq 0$, then

$$
\begin{aligned}
b_{k} & \subset \operatorname{rot}_{s}\left(\left[0, x_{1}\right] \times\left[0, x_{2}\right]\right), \quad k>0, \\
\mid \operatorname{rot}_{s}\left(\left[0, x_{1}\right]\right. & \left.\times\left[0, x_{2}\right]\right) \cap b_{k}|\leq| b_{k} \mid / 2=\pi r^{2} / 2, \quad k<0,
\end{aligned}
$$

and then similarly we obtain (2.12). Now we prove that if

$$
R \in \mathcal{R}_{s}, \quad 3 \gamma<|s|<\pi / 2-3 \gamma,
$$

then

$$
\left|\int_{R} \phi(x) d x\right| \leq \frac{10}{N}<\varepsilon^{3}
$$

We have

$$
\begin{aligned}
\int_{R} \phi(x) d x & =\frac{1}{2 \pi N r^{2}} \sum_{b_{k} \cap R \neq \emptyset} \int_{R} \mathbb{I}_{b_{k}}(x) d x \\
= & \frac{1}{2 \pi N r^{2}} \sum_{\theta_{k} \in R} \int_{R} \mathbb{I}_{b_{k}}(x) d x+\frac{1}{2 \pi N r^{2}} \sum_{\theta_{k} \notin R, b_{k} \cap R \neq \emptyset} \int_{R} \mathbb{I}_{b_{k}}(x) d x .
\end{aligned}
$$

The conditions $\theta_{k} \notin R, b_{k} \cap R \neq \emptyset$ mean that $b_{k}$ intersects a side of $R$. Also if a line $l$ contains a side of $R$ then $|\arg l|>3 \gamma$. On the other hand, by (3) any line with $|\arg l|>3 \gamma$ can intersect not more than two balls $b_{k}$. So the number of terms in the second sum does not exceed 8 . Therefore

$$
\left|\frac{1}{2 \pi N r^{2}} \sum_{\theta_{k} \notin R, b_{k} \cap R \neq \emptyset} \int_{R} \mathbb{I}_{b_{k}}(x) d x\right| \leq \frac{4}{N} .
$$

For the same reason the equality

$$
\int_{R} \mathbb{I}_{b_{k}}(x) d x=\int_{\mathbb{R}^{2}} \mathbb{I}_{b_{k}}(x) d x
$$

fails for not more than eight different $k$ 's. Therefore

$$
\left|\frac{1}{2 \pi N r^{2}} \sum_{\theta_{k} \in R} \int_{R} \mathbb{I}_{b_{k}}(x) d x-\frac{1}{2 \pi N r^{2}} \sum_{\theta_{k} \in R} \int_{\mathbb{R}^{2}} \mathbb{I}_{b_{k}}(x) d x\right| \leq \frac{4}{N} .
$$


Hence we obtain

$$
\begin{aligned}
\left|\frac{1}{2 \pi N r^{2}} \sum_{\theta_{k} \in R} \int_{R} \mathbb{I}_{b_{k}}(x) d x\right| & \leq\left|\frac{1}{2 \pi N r^{2}} \sum_{\theta_{k} \in R} \int_{\mathbb{R}^{2}} \mathbb{I}_{b_{k}}(x) d x\right|+\frac{4}{N} \\
& =\left|\frac{1}{2 N} \sum_{\theta_{k} \in R} \operatorname{sign}(k)\right|+\frac{4}{N} \leq \frac{5}{N},
\end{aligned}
$$

where the last inequality follows from Lemma 2. Combining (2.17)-(2.19) we get (2.16). Fix $s$ with $3 \gamma<|s| \leq \pi / 4$ and take a point $x \in \mathbb{R}^{2}$ such that

$$
x \notin \Gamma_{s}(2 \varepsilon) \cup \Gamma_{s^{\perp}}(2 \varepsilon), \quad x \in R \in \mathcal{R}_{s}, \quad 3 \gamma<|s|<\pi / 2-3 \gamma .
$$

We need to prove

$$
\frac{1}{|R|} \int_{R} \phi(t) d t \leq \varepsilon .
$$

Assume the lengths of the sides of $R$ are $a$ and $b$. If $R$ contains no $\theta_{k}$ then (2.20) is trivial. So suppose at least one $\theta_{k}$ is in $R$. Hence $R$ intersects $B(\varepsilon)$ and $\left(\Gamma_{s}(2 \varepsilon) \cup \Gamma_{s^{\perp}}(2 \varepsilon)\right)^{\mathrm{c}}$. As $R \in \mathcal{R}_{s}$, we get $a, b>\varepsilon$. Hence (2.16) yields

$$
\frac{1}{|R|} \int_{R} \phi(t) d t \leq \frac{\varepsilon^{3}}{a b} \leq \varepsilon .
$$

Lemma 4. For any $0<\varepsilon, \delta<1 / 10$, and any interval $S=[\alpha-\gamma, \alpha+\gamma] \subset$ $[0, \pi / 2)$ with $0<\gamma \leq \pi / 12$, there exist a bounded function $\phi$ and numbers $\nu, \nu^{\prime}$ with $0<\nu<\nu^{\prime}$ such that

$$
\sup _{k \in \mathbb{Z}^{2}} \int_{Q_{k}}|\phi(x)| d x \leq 1,
$$

$$
\begin{gathered}
\operatorname{mes}^{*}\left\{x \in \mathbb{R}^{2}: M_{s} \phi(x)>\varepsilon\right\}<\varepsilon, \quad 3 \gamma<|s-\alpha|<\pi / 2-3 \gamma, \\
\operatorname{mes}^{*}\left\{x \in \mathbb{R}^{2}: M_{s}^{[0, \nu)} \phi(x)>\varepsilon\right\}<\varepsilon, \quad s \in[0,2 \pi), \\
M_{s}^{\left[\nu^{\prime}, \infty\right)} \phi(x)<\varepsilon, \quad x \in \mathbb{R}^{2}, s \in[0,2 \pi), \\
\operatorname{mes}_{*}\left\{M_{s}^{\left[\nu, \nu^{\prime}\right]} \phi(x)>1 / \delta\right\}>\frac{\delta}{4} \ln \frac{1}{12 \delta}, \quad s \in S .
\end{gathered}
$$

Proof. Without loss of generality we may assume $\alpha=0$, i.e. $S=[-\gamma, \gamma]$. We take $\lambda=\min \{\varepsilon / 100, \delta\}$ and consider a double sequence $\varepsilon_{k}=\varepsilon_{k_{1}, k_{2}}=$ $\lambda 2^{-\left(\left|k_{1}\right|+\left|k_{2}\right|\right)}, k \in \mathbb{Z}^{2}$. Using Lemma 3 we can find functions $\phi_{k}$ satisfying the following conditions:

$$
\begin{gathered}
\operatorname{supp} \phi_{k} \subset B\left(\varepsilon_{k}\right) \subset B(\varepsilon), \\
\int_{Q_{0}} \phi_{k}(x) d x=0, \quad \int_{Q_{0}}\left|\phi_{k}(x)\right| d x \leq 1,
\end{gathered}
$$




$$
\int_{\operatorname{rot}_{s} R_{x}} \phi_{k}(x) d x>1 / 4, \quad R_{x}=\left[0, x_{1}\right] \times\left[0, x_{2}\right],
$$

$$
x_{1}, x_{2} \geq \delta \geq \varepsilon_{k},|s|<\gamma,
$$

(2.29) $M_{s} \phi_{k}(x)<\varepsilon_{k} \quad$ if $x \notin \Gamma_{s}\left(2 \varepsilon_{k}\right) \cup \Gamma_{s^{\perp}}\left(2 \varepsilon_{k}\right), 3 \gamma<|s| \leq \pi / 2-3 \gamma$,

where $k=\left(k_{1}, k_{2}\right)$. Define

$$
\begin{gathered}
\phi(x)=\sum_{k \in \mathbb{Z}^{2}} \phi_{k}(x+k), \\
E_{s}=\bigcup_{k \in \mathbb{Z}^{2}}\left(k+\left(\Gamma_{s}\left(2 \varepsilon_{k}\right) \cup \Gamma_{s^{\perp}}\left(2 \varepsilon_{k}\right)\right)\right) .
\end{gathered}
$$

We obviously have (2.21) and

$$
\begin{aligned}
& \operatorname{supp} \phi \subset \bigcup_{k \in \mathbb{Z}^{2}}(k+B(\varepsilon)), \\
& \int_{Q_{k}} \phi(x) d x=0, \quad k \in \mathbb{Z}^{2} .
\end{aligned}
$$

Proof of (2.22): For any square $Q_{j}, j \in \mathbb{Z}^{2}$, we have

$$
\begin{gathered}
\left|Q_{j} \cap\left(k+\Gamma_{s}\left(2 \varepsilon_{k}\right)\right)\right| \leq \operatorname{diam} Q_{j} \times \operatorname{mes}_{s}\left(k+\Gamma_{s}\left(2 \varepsilon_{k}\right)\right)=4 \varepsilon_{k} \sqrt{2}, \\
\left|Q_{j} \cap\left(k+\Gamma_{s^{\perp}}\left(2 \varepsilon_{k}\right)\right)\right| \leq 4 \varepsilon_{k} \sqrt{2} .
\end{gathered}
$$

Hence we obtain

$$
\text { mes }^{*} E_{s} \leq \sum_{k} 8 \sqrt{2} \varepsilon_{k}=32 \sqrt{2} \lambda \leq \varepsilon .
$$

From (2.29) it follows that

$$
M_{s} \phi_{k}(x+k) \leq \varepsilon_{k}, \quad x \notin E_{s} \supset k+\left(\Gamma_{s}\left(2 \varepsilon_{k}\right) \cup \Gamma_{s^{\perp}}\left(2 \varepsilon_{k}\right)\right), 3 \gamma<|s| \leq \pi / 2-3 \gamma .
$$

Then according to (2.30) and (2.31) we get

$$
M_{s} \phi(x) \leq \sum_{k} M_{s} \phi_{k}(x+k) \leq \sum_{k} \varepsilon_{k} \leq \varepsilon, \quad x \notin E_{s}, 3 \gamma<|s| \leq \pi / 2-3 \gamma,
$$

and combining this with (2.34) we obtain (2.22).

Proof of (2.23): From (2.32) it follows that

$$
\lim _{\nu \rightarrow 0} M_{s}^{[0, \nu)} \phi(x)=0 \quad \text { if } x \notin \bigcup_{k \in \mathbb{Z}^{2}}(k+B(\varepsilon)), s \in[0,2 \pi),
$$

therefore for small $\nu<\delta$ we have (2.23), since

$$
\operatorname{mes}^{*}\left(\bigcup_{k \in \mathbb{Z}^{2}}(k+B(\varepsilon))\right)=|B(\varepsilon)|=\pi \varepsilon^{2} \leq \varepsilon
$$


Proof of (2.24): From (2.33) we obtain

$$
\lim _{\nu^{\prime} \rightarrow \infty} \int_{R} \phi\left(\nu^{\prime} x\right) d x=0
$$

for any rectangle $R$, and the convergence is uniform in $R \in \mathcal{R}_{s}^{[1, \infty)}, s \in$ $[0,2 \pi)$. So for large $\nu^{\prime}>1 / 4$ we have

$$
M_{s}^{[1, \infty)} \phi\left(\nu^{\prime} x\right)<\varepsilon, \quad x \in \mathbb{R}^{2}, s \in[0,2 \pi) .
$$

By dilation we get

$$
M_{s}^{\left[\nu^{\prime}, \infty\right)} \phi(x)=M_{s}^{[1, \infty)} \phi\left(\nu^{\prime} x\right)<\varepsilon, \quad x \in \mathbb{R}^{2}, s \in[0,2 \pi),
$$

which gives (2.24).

Proof of (2.25): Consider the set

$$
A=\left\{x=\left(x_{1}, x_{2}\right): x_{1} x_{2} \leq \delta / 4, \delta \leq x_{1}, x_{2} \leq 1 / 4\right\} .
$$

We have

$$
\begin{gathered}
\operatorname{rot}_{s} A \subset B(1 / 2), \quad s \in[-\pi / 4, \pi / 4), \\
|A|=\int_{\delta}^{1 / 4} \frac{\delta}{4 t} d t-\delta(1 / 4-\delta)>\frac{\delta}{4} \ln \frac{1}{12 \delta} .
\end{gathered}
$$

If $x=\left(x_{1}, x_{2}\right) \in A$, then

$$
1 / 4 \geq x_{1}, x_{2} \geq \delta>\varepsilon_{k}, \quad\left|R_{x}\right| \leq \delta / 4
$$

So by $(2.28)$,

$$
\int_{k+\operatorname{rot}_{s} R_{x}} \phi_{k}(k+t) d t=\int_{\operatorname{rot}_{s} R_{x}} \phi_{k}(t) d t>1 / 4 \quad \text { if } x \in A,|s|<\gamma,
$$

and therefore from (2.26) we get

$$
\int_{k+\operatorname{rot}_{s} R_{x}} \phi(t) d t=\int_{k+\operatorname{rot}_{s} R_{x}} \phi_{k}(k+t) d t>1 / 4 \quad \text { if } x \in A,|s|<\gamma .
$$

As $\nu<\delta, \nu^{\prime}>1 / 4$ we have $R_{x} \in \mathcal{R}_{0}^{[\delta, 1 / 4]} \subset \mathcal{R}_{0}^{\left[\nu, \nu^{\prime}\right]}$. Since $\left|R_{x}\right| \leq \delta / 4$, from (2.39) and (2.38) we conclude that

$$
M_{s}^{\left[\nu, \nu^{\prime}\right]} \phi(x)>\frac{1}{4\left|R_{x}\right|}>\frac{1}{\delta}, \quad x \in G_{s}=\bigcup_{k}\left(k+\operatorname{rot}_{s} A\right),|s|<\gamma .
$$

In addition, by (2.35)-(2.37), for any $m \in \mathbb{Z}^{2}$ we get

$$
\left|\left(m+Q_{0}\right) \cap G_{s}\right|=\left|m+\operatorname{rot}_{s} A\right|=|A|>\frac{\delta}{4} \ln \frac{1}{12 \delta},
$$

which implies

$$
\text { mes }^{*} G_{s}>\frac{\delta}{4} \ln \frac{1}{12 \delta} .
$$

Combining this with (2.40) we obtain (2.25). 


\section{Proofs of theorems}

Proof of Theorem 1. Let $G$ be an arbitrary $G_{\delta}$-set in $[0, \pi / 2)$. So

$$
G=\left(\bigcap_{k=1}^{\infty} G_{k}\right) \cap[0, \pi / 2),
$$

where $G_{k} \subset \mathbb{R}$ are open sets and $G_{1} \supseteq \cdots \supseteq G_{n} \supseteq \cdots$. Each $G_{k}$ is a union $G_{k}=\bigcup_{j} I_{j}^{k}$ of mutually disjoint intervals.

We note that an arbitrary interval $I=(\alpha, \beta) \subset \mathbb{R}$ can be split into disjoint intervals $I_{i}=\left[\alpha_{i}, \beta_{i}\right)$ such that

$$
\left|I_{i}\right| \leq \pi / 12, \quad 3 I_{i} \subset I, \quad \sum_{i} \mathbb{I}_{3 I_{i}}(x) \leq 8 .
$$

For $I=(-1,1)$ such a partition is

$$
\begin{array}{ll}
{\left[1-(9 / 10)^{k}, 1-(9 / 10)^{k+1}\right),} & k=0,1,2, \ldots, \\
{\left[(9 / 10)^{k+1}-1,(9 / 10)^{k}-1\right),} & k=0,1,2, \ldots
\end{array}
$$

We do a similar splitting for any $I_{j}^{k}$. Let $J_{t}, t=1,2, \ldots$, be an enumeration of those splitting intervals $J$ for which $J \cap[0, \pi / 2) \neq \emptyset$. We set $l_{t}=J_{t} \cap[0, \pi / 2)$. It is easy to check the following:

(1) if $x \in G$, then $x$ belongs to an infinite number of $l_{t}$ 's,

(2) if $x \notin G$ then $x$ belongs only to a finite number of $3 l_{t}$ 's.

We choose integers $0=m_{0}<m_{1}<m_{2}<\cdots$ satisfying

$$
\prod_{k=m_{t}+1}^{m_{t+1}}\left(1-\frac{1}{k \ln k}\right)<\frac{1}{2^{t}}, \quad t=1,2, \ldots
$$

We define

$$
S_{k}=l_{t} \quad \text { if } m_{t}<k \leq m_{t+1} .
$$

Using Lemma 4 for $S=S_{k}, \varepsilon=1 / 2^{k}, \delta=1 /\left(k \ln ^{2} k\right)$, we may define functions $\phi_{k}$ and numbers $0<\nu_{k}<\nu_{k}^{\prime}$ satisfying (2.21)-(2.25). We set

$$
\begin{aligned}
U_{s, k} & =\left\{x \in \mathbb{R}^{2}: M_{s} \phi_{k}(x) \leq 1 / 2^{k}\right\}, \\
V_{s, k}^{\prime} & =\left\{x \in \mathbb{R}^{2}: M_{s}^{\left[0, \nu_{k}\right)} \phi_{k}(x) \leq 1 / 2^{k}\right\}, \\
V_{s, k}^{\prime \prime} & =\left\{x \in \mathbb{R}^{2}: M_{s}^{\left[\nu_{k}, \nu_{k}^{\prime}\right]} \phi_{k}(x)>k \ln ^{2} k\right\} .
\end{aligned}
$$

By (2.22), (2.23), (2.25) we have

$$
\operatorname{mes}_{*} U_{s, k}>1-1 / 2^{k}, \quad s \in[0, \pi / 2) \backslash 3 S_{k}
$$

(we may replace the condition $3 \gamma<|s-\alpha|<\pi / 2-3 \gamma$ in (2.22) by $s \in$ 
$[0, \pi / 2) \backslash 3 S$ because the latter implies the former) and

(3.8) $\quad \operatorname{mes}_{*} V_{s, k}^{\prime \prime}>\frac{1}{4 k \ln ^{2} k} \ln \frac{k \ln ^{2} k}{12}>\frac{c}{k \ln k}, \quad s \in S_{k}(k \geq 3)$.

From (2.24) we get

$$
M_{s}^{\left[\nu_{k}^{\prime}, \infty\right)} \phi_{k}(x)<1 / 2^{k}, \quad x \in \mathbb{R}^{2}, s \in[0, \pi / 2) .
$$

We define integers $1=n_{0}<n_{1}<n_{2}<\cdots$ so that

$$
\frac{n_{k}}{n_{k-1}}>\max \left(\frac{4}{\nu_{k-1}}, \frac{\nu_{k}^{\prime}}{\nu_{k-1}}\right), \quad k=1,2, \ldots,
$$

and set $\mu_{k}=\nu_{k} / n_{k}$. It is clear that

$$
\mu_{k-1}>\nu_{k}^{\prime} / n_{k}>\mu_{k}, \quad k=2,3, \ldots
$$

Consider the functions

$$
\psi_{k}(x)=\phi_{k}\left(n_{k} x\right), \quad x \in Q_{0} .
$$

According to (3.3)-(3.5) and (3.11), we obviously have

$$
\begin{gathered}
(3.12) \quad M_{s} \psi_{k}(x) \leq 1 / 2^{k}, \quad x \in \operatorname{dil}_{n_{k}} U_{s, k}, s \in[0, \pi / 2) \backslash 3 S_{k}, \\
(3.13) \quad M_{s}^{\left[0, \mu_{k}\right)} \psi_{k}(x)=M_{s}^{\left[0, \nu_{k} / n_{k}\right)} \psi_{k}(x) \leq 1 / 2^{k}, \\
\quad x \in \operatorname{dil}_{n_{k}} V_{s, k}^{\prime}, s \in[0, \pi / 2), \\
(3.14) \quad M_{s}^{\left[\mu_{k}, \mu_{k-1}\right]} \psi_{k}(x)>M_{s}^{\left[\nu_{k} / n_{k}, \nu_{k}^{\prime} / n_{k}\right]} \psi_{k}(x)>k \ln ^{2} k, \\
\quad x \in \operatorname{dil}_{n_{k}} V_{s, k}^{\prime \prime}, s \in S_{k}, \\
\text { (3.15) } \quad M_{s}^{\left[\mu_{k-1}, \infty\right)} \psi_{k}(x) \leq M_{s}^{\left[\nu_{k}^{\prime} / n_{k}, \infty\right)} \psi_{k}(x) \leq 1 / 2^{k}, \quad x \in \mathbb{R}^{2}, s \in[0, \pi / 2) .
\end{gathered}
$$

The desired function is

$$
f(x)=\sum_{k=1}^{\infty} \frac{\psi_{k}(x)}{k \ln ^{3 / 2} k}, \quad x \in Q_{0} .
$$

Set

$$
U_{s}=\limsup _{k \rightarrow \infty}\left(\left(\operatorname{dil}_{n_{k}} U_{s, k}\right) \cap Q_{0}\right),
$$

where $\lim \sup _{k \rightarrow \infty} A_{k}$ means $\bigcup_{n} \bigcap_{k \geq n} A_{k}$. If $s \notin G$, then (2) implies $s \in$ $[0, \pi / 2) \backslash 3 S_{k}$ for $k>k(s)$. Therefore, by (3.6) we have $\left|\operatorname{dil}_{n_{k}} U_{s, k} \cap Q_{0}\right| \geq$ $\operatorname{mes}_{*} U_{s, k}>1-1 / 2^{k}, k>k(s)$, and so we get

$$
\left|U_{s}\right|=1 \quad \text { if } s \notin G \text {. }
$$

From (3.12) and (3.17) we infer that for any $x \in U_{s}$,

$$
M_{s} \psi_{k}(x) \leq 1 / 2^{k}, \quad k>k(x) .
$$


Hence, if $\varepsilon>0$, then for an appropriate $N>k(x)$ we get

$$
M_{s}\left(\sum_{k=N+1}^{\infty} \frac{\psi_{k}(x)}{k \ln ^{3 / 2} k}\right) \leq \sum_{k=N+1}^{\infty} \frac{M_{s} \psi_{k}(x)}{k \ln ^{3 / 2} k} \leq \sum_{k=N+1}^{\infty} \frac{1}{k 2^{k} \ln ^{3 / 2} k}<\varepsilon
$$

On the other hand, since

$$
\sum_{k=1}^{N} \frac{\psi_{k}(x)}{k \ln ^{3 / 2} k}
$$

is a bounded function, the basis $\mathcal{R}_{s}$ differentiates its integral. So, by (3.19) and (3.16), $\mathcal{R}_{s}$ differentiates $\int f$ if $s \in[0, \pi / 2) \backslash G$.

Now let $s \in G$. Then $s \in l_{t_{i}}, i=1,2, \ldots$ Hence $s \in S_{k}$ if $m_{t_{i}}<k \leq$ $m_{t_{i}+1}, i=1,2, \ldots$ We notice that each $V_{s, k}^{\prime \prime}$ defined in (3.5) is a $\nu_{k}$-set, and by (3.10), $n_{k+1}>4 n_{k} / \nu_{k}$. Therefore, using (3.1), from Lemma 1 we obtain

$$
\left|\bigcup_{k=m_{t_{i}}+1}^{m_{t_{i}+1}} \operatorname{dil}_{n_{k}} V_{s, k}^{\prime \prime} \cap Q_{0}\right| \geq 1-\prod_{k=m_{t_{i}}+1}^{m_{t_{i}+1}}\left(1-\frac{1}{k \ln k}\right)>1-\frac{1}{2^{t}} .
$$

Defining

$$
V_{s}=\left(\limsup _{k \rightarrow \infty} \operatorname{dil}_{n_{k}} V_{s, k}^{\prime}\right) \cap\left(\limsup _{i \rightarrow \infty} \bigcup_{k=m_{t_{i}}+1}^{m_{t_{i}+1}} \operatorname{dil}_{n_{k}} V_{s, k}^{\prime \prime}\right) \cap Q_{0},
$$

from (3.20) and (3.7) we get

$$
\left|V_{s}\right|=1, \quad s \in G .
$$

On the other hand, if $x \in V_{s}$, then

$$
\begin{aligned}
& x \in \operatorname{dil}_{n_{k_{i}}} V_{s, k_{i}}^{\prime \prime}, \quad i=1,2, \ldots, \\
& x \in \operatorname{dil}_{n_{k}} V_{s, k}^{\prime}, \quad k>k(x),
\end{aligned}
$$

where $k_{i} \rightarrow \infty$, and therefore, by (3.13) and (3.15) we have

$$
M_{s}^{\left[\mu_{k_{i}}, \mu_{k_{i}-1}\right]} \psi_{j}(x) \leq 1 / 2^{k_{i}} \quad \text { if } j \neq k_{i} .
$$

The case $j>k_{i}$ follows from (3.15), and $j<k_{i}$ from (3.13). From (3.14) we get

$$
M_{s}^{\left[\mu_{k_{i}}, \mu_{\left.k_{i}-1\right]}\right]} \psi_{k_{i}}(x)>k_{i} \ln ^{2} k_{i} .
$$

So if $k_{i}>k(x)$, then

$$
\begin{aligned}
& M_{s} f(x) \geq M_{s}^{\left[\mu_{k_{i}}, \mu_{k_{i}-1}\right]} f(x) \\
& \quad \geq \frac{M_{s}^{\left[\mu_{k_{i}}, \mu_{k_{i}-1}\right]} \psi_{k_{i}}(x)}{k_{i} \ln ^{3 / 2} k_{i}}-\sum_{j \neq k_{i}} \frac{M_{s}^{\left[\mu_{k_{i}}, \mu_{k_{i}-1}\right]} \psi_{j}(x)}{j \ln ^{3 / 2} j} \geq c \sqrt{\ln k_{i}}-\sum_{j \neq k_{i}} \frac{1}{j 2^{j} \ln ^{3 / 2} j},
\end{aligned}
$$

and so $\bar{D}_{s} f(x)=\infty$ whenever $x \in V_{s}$ and $s \in G$. Since $\left|V_{s}\right|=1$ by (3.21), the theorem is completely proved. 
Proof of Theorem 2. The necessity is shown in the introduction. To prove the sufficiency we let $V \subset[0, \pi / 2)$ be an arbitrary $G_{\delta \sigma}$-set,

$$
V=\bigcup_{n} V_{n}
$$

where each $V_{n}$ is a $G_{\delta}$. According to Theorem 1 for each $V_{n}$ there exists a function $f_{n} \in L^{1}\left(\mathbb{R}^{2}\right)$ such that its integral is differentiated by $\mathcal{R}_{s}$ if $s \notin V_{n}$, and $\bar{D}_{s} f_{n}(x)=\infty$ a.e. if $s \in V_{n}$. Set $g_{n}(x)=\mathbb{I}_{Q_{n}}(x) f_{n}(x)$, where $Q_{n}$ is a family of arbitrary pairwise disjoint unit open squares, and consider the function

$$
g(x)=\sum_{n=1}^{\infty} g_{n}(x) .
$$

Since the supports of the functions $g_{n}$ are disjoint, for any $x \in Q_{n}$ and any $s$ we have

$$
\bar{D}_{s} g(x)=\bar{D}_{s} g_{n}(x)=\bar{D}_{s} f_{n}(x) \text {. }
$$

If $s \in V$ then $s \in V_{n}$ for some $n$. So we get $\bar{D}_{s} g(x)=\bar{D}_{s} f_{n}(x)=\infty$ almost everywhere on the square $Q_{n}$. Using disjointness of the supports of the functions $g_{n}$ once again we conclude that if $s \notin V$ then

$$
\lim _{\operatorname{diam}(R) \rightarrow 0, x \in R \in \mathcal{R}_{s}} \frac{1}{|R|} \int_{R} g=g(x) \quad \text { a.e. }
$$

Finally, we conclude that $V$ is a $W R$-set and Theorem 2 is proved.

\section{References}

[1] Y. El Helou, Recouvrement du tore $T^{d}$ par des ouverts aléatoires, Thèse, Univ. Paris (Orsay), 1978.

[2] M. de Guzmán, Differentiation of Integrals in $\mathbb{R}^{n}$, Lecture Notes in Math. 481, Springer, Berlin, 1975.

[3] B. Jessen, J. Marcinkiewicz and A. Zygmund, Note on the differentiability of multiple integrals, Fund. Math. 25 (1935), 217-237.

[4] G. Lepsveridze, On strong differentiability of integrals along different directions, Georgian Math. J. 2 (1995), 613-630.

[5] - , To the problem of a strong differentiability of integrals along different directions, ibid. 5 (1998), 157-176.

[6] B. López Melero, A negative result in differentiation theory, Studia Math. 72 (1982), 173-182.

[7] J. Marstrand, A counter-example in the theory of strong differentiation, Bull. London Math. Soc. 9 (1977), 209-211.

[8] G. G. Oniani, On the differentiation of integrals with respect to the bases $B_{2}(\theta)$, East J. Approx. 3 (1997), 275-301.

[9] —, Differentiation of Lebesgue integrals, Univ. of Tbilisi, Tbilisi, 1998 (in Russian).

[10] - On the strong differentiation of multiple integrals along different frames, II, Proc. A. Razmadze Math. Inst. 126 (2001), 122-125. 
[11] G. G. Oniani, On the strong differentiation of multiple integrals along different frames, Georgian Math. J. 12 (2005), 349-368.

[12] S. Saks, Remark on the differentiability of the Lebesgue indefinite integral, Fund. Math. 22 (1934), 257-261.

[13] A. M. Stokolos, An inequality for equimeasurable rearrangements and its application in the theory of differentiation of integrals, Anal. Math. 9 (1983), 133-146.

[14] —, On a problem of A. Zygmund, Math. Notes 64 (1998), 646-657.

Department of Computer Science

Yerevan State University

and

Institute of Mathematics

Armenian National Academy of Sciences

Marshal Baghramian ave. 24b

Yerevan, 375019, Armenia

E-mail: karagul@instmath.sci.am

Received January 10, 2006

Revised version June 7, 2007 